\title{
The Design and Implementation of Smart Trash Bin
}

\author{
Fady E. F. Samann \\ College of Engineering, Department of Computer and Communication, Nawroz University, Duhok, Kurdistan \\ Region - Iraq
}

\begin{abstract}
This paper presents a cost-effective design of an intelligent waste container for small-scale cases. This system is based on Arduino Nano board and an ultrasonic sensor to monitor the fullness level of the container and give SMS alerts using a GSM module. The system is powered by lithium battery power bank supported by solar cell panel. The system provides an option of charging external portable devices using the power bank. Moreover, the system will store usage events, recorded by PIR sensor, and fullness events on a memory card, which is also used to play audio message using a speaker, when the bin is being used. Finally, the system is implemented successfully with an acceptable overall cost for the intended application. The system performance was found satisfactory according to the obtained test results.
\end{abstract}

KEYWORDS: Smart Device, Trash Bin, Waste Container, Microcontroller, GSM, Ultrasonic Sensor.

\section{INTRODUCTION}

Environmental problems are raised by modern cities for waste collection and disposal[1]. Therefore, smart waste management systems became essential for cities that aim to reduce cost and manage resources and time[2]. Currently, the trend is shifting towards smart devices and internet of things (IoT) solutions to overcome common problems such as waste management issues[3]. Optimizing the process of trash collection is the main purpose of the smart solutions provided by industry. However, the cost of applying such solutions is still relatively high [4]. The purpose of this work is to present a cost-effective smart trash bin for localized and smallscale cases, such as small parks, university campus and hospitals. The literature of this paper will present a literature review of past related papers and commercial solutions. Then methodology and methods section will explain the work of the system and all the hardware and software used in this work, besides the design of the smart trash bin. Finally, the results of tests will be discussed followed by conclusions and future work.

Academic Journal of Nawroz University (AJNU)

Volume 6, No 3(2017), 8 pages

Received 1 May 2017; Accepted 16 August 2017

Regular research paper: Published 30 August 2017

Corresponding author's e-mail: Fady.fathel@nawroz.edu.krd

Copyright $@ 2017$ Fady E. F. Samann

This is an open access article distributed under the Creative Commons Attribution License.

\section{Literature Review}

\subsection{Research Papers}

The most current related work is done by Zavare and his colleagues[5] on sensor nodes connected to an Arduino board based control station, that uses a GSM module to send the sensor nodes data by SMS to the garbage collecting vehicle and to a server hosting web application by a Wi-Fi connection. The sensor nodes of the smart bins rely on the ultrasonic sensor to sense the fullness percentage according to pre-calculated bin depth. Moreover, a GPS module is used to get the bin location. The GPS module and the ultrasonic sensor are controlled by Amica R2 NodeMCU microcontroller board which has a built-in Wi-Fi module, that is used to connect to the control station.

Another work on wireless sensor network is done by Singh, Mahajan and Bagai[6]. The bins in his work are equipped with an accelerometer sensor to sense the opening and closing of the bin lid, a temperature and humidity sensor to check the present organic waste, and an ultrasonic sensor to sense the fullness status of the bin. All these sensors are controlled by Zigbee Pro microcontroller board, which has a built-in Wi-Fi module that is used to send the sensors data to a gateway. This paper also used the same type of microcontroller board in the gateway to receive the bins data and send it to a control station, that contains a server, over GPRS. The server in the control station relies on Caspio database management system with a web based user interface.

A paper by Navghane, Killedar and Rohokale[7] examined the use of weight sensor and three IR sensors to check the 
fullness status of the smart bin and send the sensors data to a web page over Wi-Fi network to a mobile phone. The microcontroller board used in this paper was ARM LPC2148.

A report was done by students of California Polytechnic State University [8], thoroughly exploited the economic and power consumption aspects of converting a conventional outdoor trash bin into a smart one. According to the literature, the project is based on u-blox C027-U20 microcontroller board, which has built-in GPS module and cellular module. The board is used to control HC-SR04 ultrasonic sensor, that measures the bin's fullness-level, and a temperature sensor for monitoring weather conditions and fire alerts. The setup is contained by $2 \times 4 \times 6$ plastic box and powered by a $12 \mathrm{~V}$ rechargeable lead-acid battery. The report mentioned that the system generates an HTTP POST request using the data from the sensors and send it to a web application, which is built using Python and Flask framework on top of an SQLite database. The web application receives the HTTP request and check if the bin is full then send SMS message using Twilio service. Moreover, Leaflet JavaScript library is used to virtualize the collected data on a map. In summary, most of the papers above did not focus on covering the overall cost and power consumption of the system, which are the main issues tackled by this paper.

\subsection{Commercial and Industrial Solutions}

There are several companies offer smart trash bins managed by a web-based application. ECUBE labs [9] and Bigbelly[10] offer smart trash compactor bins, which powered by solar cell panel and battery. Clean CUBE bin uses ultrasonic sensor and Bigbelly smart trash bin uses laser sensor to measure fullness status[9][10]. Moreover, most companies offer IoT sensors, which can be easily installed on available trash bins. ECUBE labs[9], ENEVO[11] and SMARTBIN[12] offer battery powered versions of these ultrasonic IoT sensors. Moreover, CUBE labs offer solar powered one. However, COMPOLOGY[13] offers IoT sensor that uses a camera to detect the fullness status of large industrial trash containers. Most of these IoT sensors and smart bins integrate temperature, tilt and acceleration sensors to detect vandalism, fire, trash collecting and usage events. All these solutions make use of cellular networks to send data from the IoT sensors and bins to their cloud hosted web-application portal over the internet. These web-applications monitor fullness level, energy usage, fire alerts, and give real-time readings and historical reports in addition to schedules and routes for optimized trash collection. Finally, contrary to the mentioned solutions, this paper aims to reduce cost by sending fullness alert without the need for internet connection and web-applications.

\section{Methodology and Methods}

This work will put a design for the smart trash bin, then explain the used hardware parts and how it is connected together. The software is then explained and illustrated as a flowchart. Figure 1 shows the basic operation of the system. The fullness status of the bin is determined by calculating the distance between the lid of the bin and the trash by using a sensor. A distance threshold will be set according to the bin dimensions. When the distance measuring sensor indicates that the bin is full, then a microcontroller board will control a GSM module to send SMS alert, that contains bin ID and alert message, to a predefined phone number. The location of the bin is predefined by a sanitary worker who will identify the filled bin by its ID, which received by the SMS alert. The system will return to default operation when the bin is emptied by the sanitary worker. An LED will keep blinking until the bin emptied from trash. A memory card will register all the usage and fullness alerts for later analyses. Moreover, a motion sensor will be used to detect the usage event to play a thanking audio message stored on the memory card using a speaker to encourage the bin user. A block diagram of the system is shown in Figure 2.

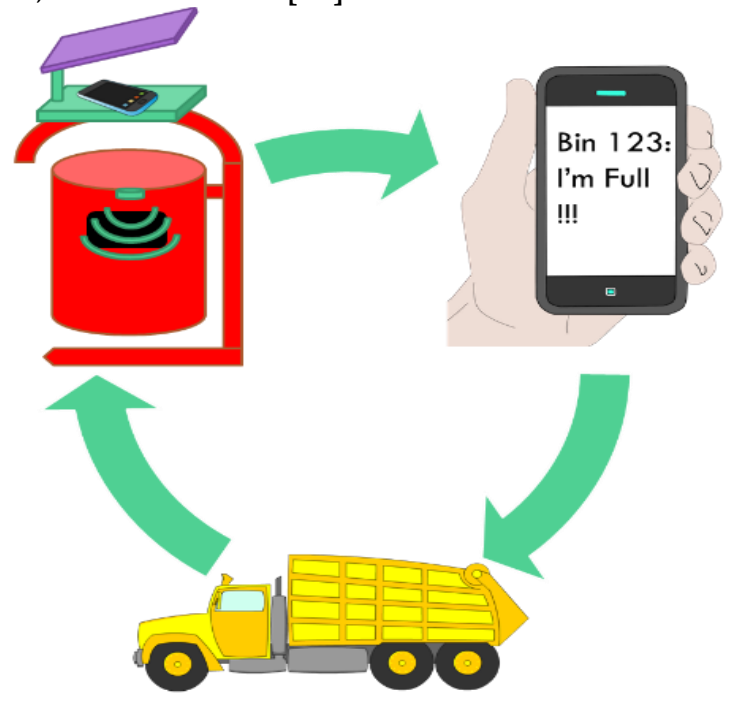

Fig 1: Basic operation of the system 


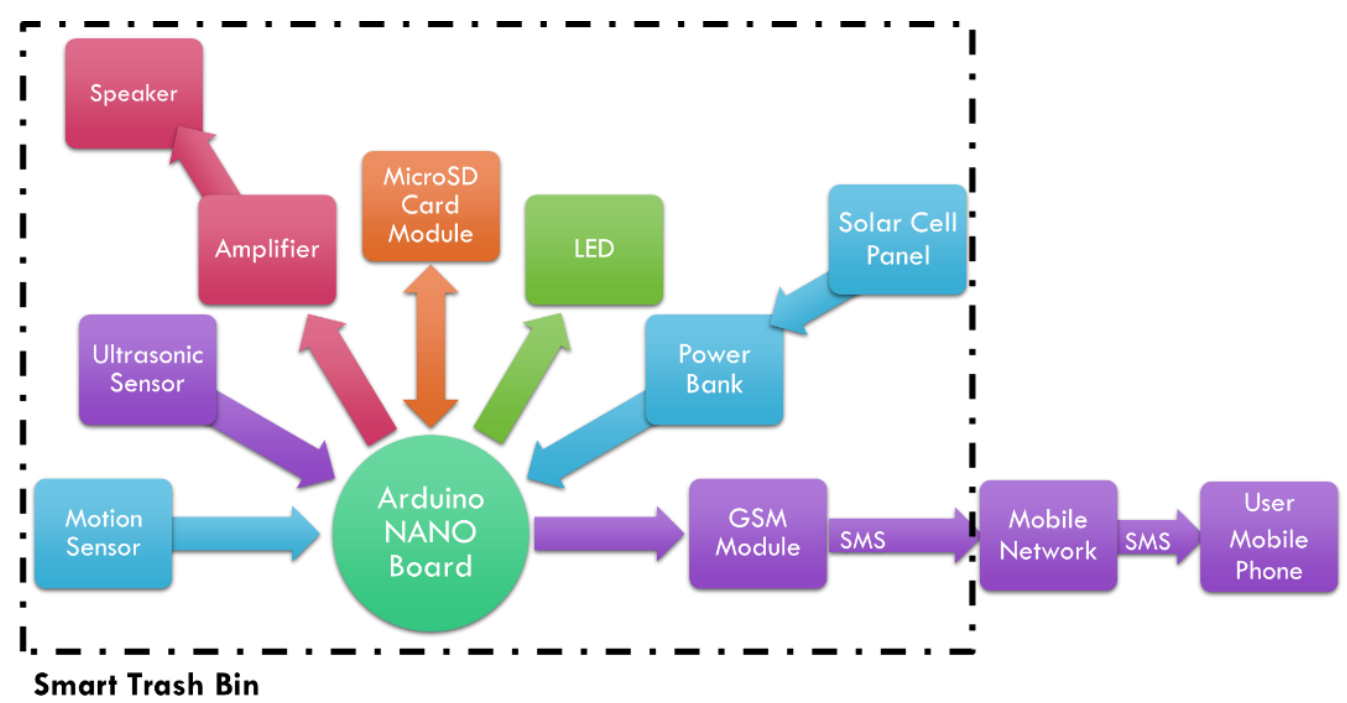

Fig 2: System Block Diagram

\subsection{Design}

The system design tries to be cost-effective and userfriendly. Figure 3 shows an outdoor trash bin after and before applying the metal work. The design relied on a commonly used outdoor trash bin, which is redesigned to append an extension arm to hold the solar cell panel. The metal work also included adding an $18 \mathrm{~cm} \times 22 \mathrm{~cm}$ tray for holding any electronic device during charging from the USB port, which will be attached to the extension arm. The height of the bin from the ground to the end of solar cell panel is $155 \mathrm{~cm}$. The trash container has a cylindrical shape of $30 \mathrm{~cm}$ diameter and $46 \mathrm{~cm}$ height. However, the height from the bottom of the bin opening is $27 \mathrm{~cm}$, which gives a volume of $76341 \mathrm{~cm}^{3}$. Moreover, all the electronic parts will be mentioned in the next section are held inside $(110 \times 180 \times 77 \mathrm{~mm})$ plastic electric junction box, which held underneath the bin lid. This design is applicable to almost any standard outdoor trash bin.

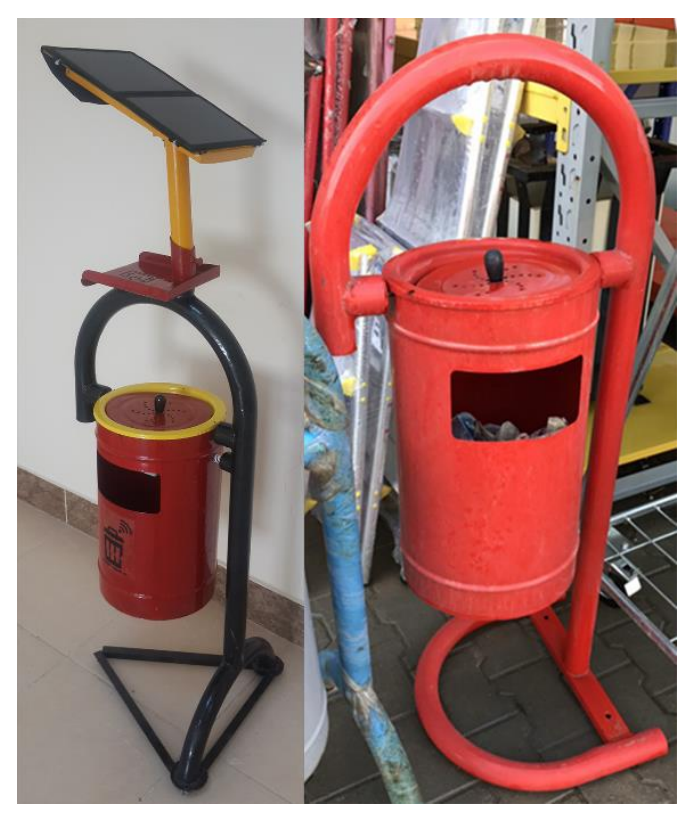

Fig 3: The design of smart trash bin, before (right) and after (left) the metal work

\subsection{Hardware}

The system structure relies on Arduino Nano board. According to the datasheet, it is based on ATmega328 microcontroller which has a $16 \mathrm{MHz}$ clock speed, $32 \mathrm{~KB}$ flash memory, $2 \mathrm{~KB}$ SRAM and $1 \mathrm{~KB}$ EEPROM. Arduino Nano is a microcontroller breadboard with integrated $5 \mathrm{~V}$ voltage regulator and can provide serial communication over USB with a computer for programming. It also has 14 digital I/O pins; 6 of them can provide PWM output and 2 external interrupt pins. This microcontroller supports SPI and I2C communications. Moreover, it also has 8 analog I/O pins. All these pins can 
deliver or accept a maximum of $40 \mathrm{~mA}$ and has an internal pull-up resistor $20-50 \mathrm{k} \Omega$. All the above comes in a small package of $18 \times 45 \mathrm{~mm}$ and weighs $4 \mathrm{~g}$. This microcontroller breadboard was chosen for its size, weight, functionality and its programming flexibility. Figure 4 and Figure 5 shows the system schematic of the circuit board and how the following electronic parts are connected inside the electric junction box. Ultrasonic ranging module sensor (HC-SR04) is used to detect the fullness level of the trash bin. According to the datasheet, this sensor can detect a $0.5 \mathrm{~m}^{2}$ object from a range of 20 $400 \mathrm{~cm}$ with a 15 -degree measuring angle. Moreover, it can detect liquid and solid objects, and also immune to almost any outdoor interference sources. This sensor returns Time of Flight $(\mathrm{ToF})$ which is the time interval that ultrasonic wave takes to cross back and forward between the wave source and the material boundary[14].

$$
\text { Distance }=\frac{\text { ToF } \times \text { Speed of Sound }}{2}
$$

The system depends on GSM module (sim900a mini v3.8.2) to send SMS fullness alerts. according to the datasheet, the module can be controlled by sending AT commands over its $5 \mathrm{~V}$ serial port. The Rx pin of GSM module is connected to analog pin A3 on the Arduino
Nano, and the Tx pin of the module is connected to A4 pin of the Arduino board. A3 and A4 pins will be turned into $\mathrm{Tx} / \mathrm{Rx}$ pins using a software library, because of the GSM module relies on serial communication and Arduino Nano has no extra serial port. An LED, with a $1 \mathrm{k} \Omega$ resistor, is used to give a visual alert when the bin is full. Moreover, a PIR motion detector (HC-SR501) is used to sense when the trash bin is being used. According to the datasheet, this sensor has a sensing range of 120 degrees within 7 meters. Therefore, the sensor is installed to the side of the plastic box and partially covered to sense only user hand entering the bin. The usage event is triggered by the PIR sensor. This sensor will interrupt the microcontroller work using pin 3 to play a WAV file stored on a MicroSD card, which is connected to the setup by an adapter from Waveshare. The audio message is played over $3 \mathrm{~W} / 4 \Omega$ speaker driven by an HXJ8002 audio amplifier. The MicroSD card is also used to log the fullness and usage events inside CSV files for further analyses. Finally, the setup of the system is powered by off-the-shelf $12000 \mathrm{mAh}$ power bank, which will also be used to charge any electronic device provided by bin user. The power bank is backed up by $13 \mathrm{~W} / 5 \mathrm{~V}$ solar cell panel which can supply current up to 2.6A.

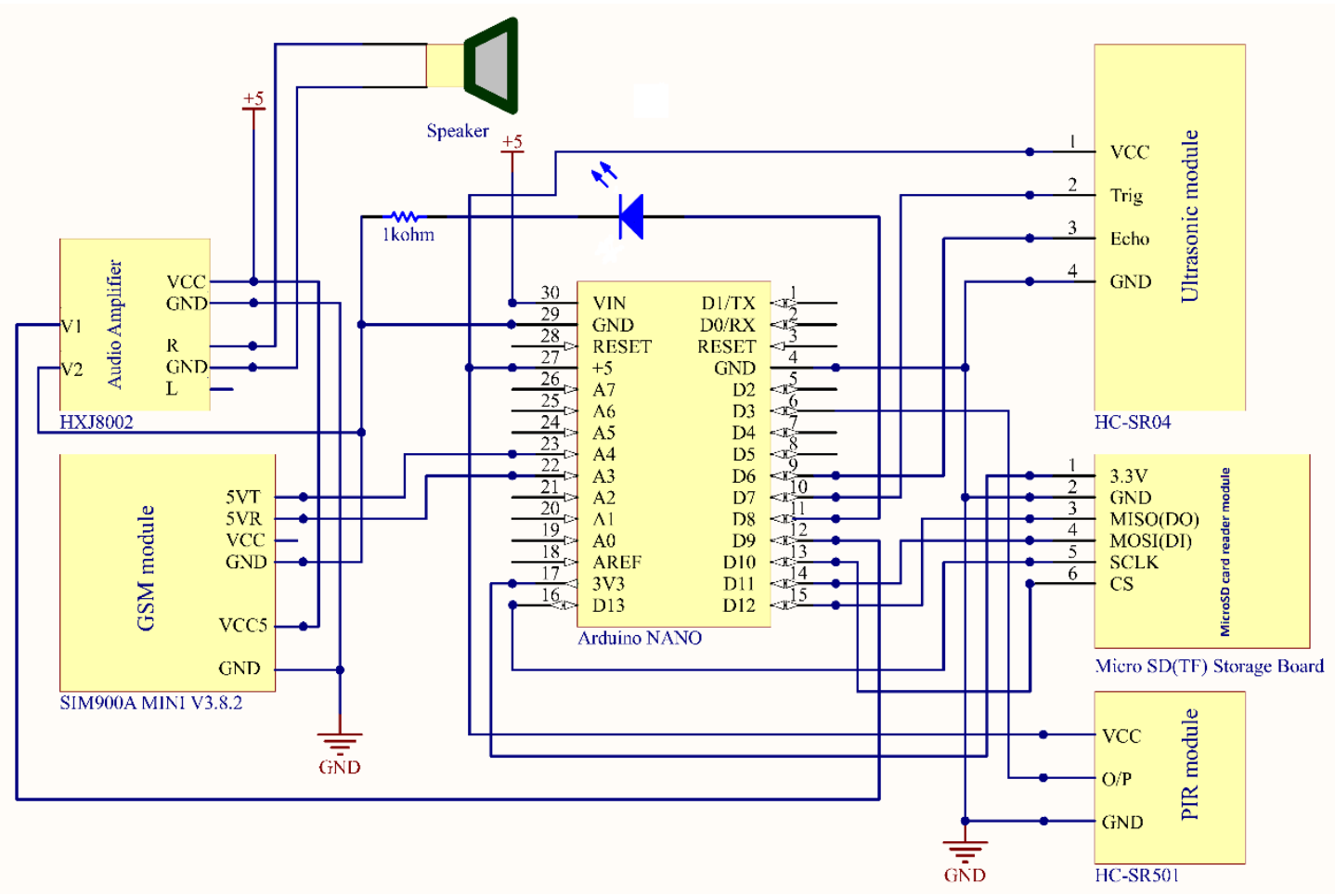

Fig 4: Circuit board schematic 


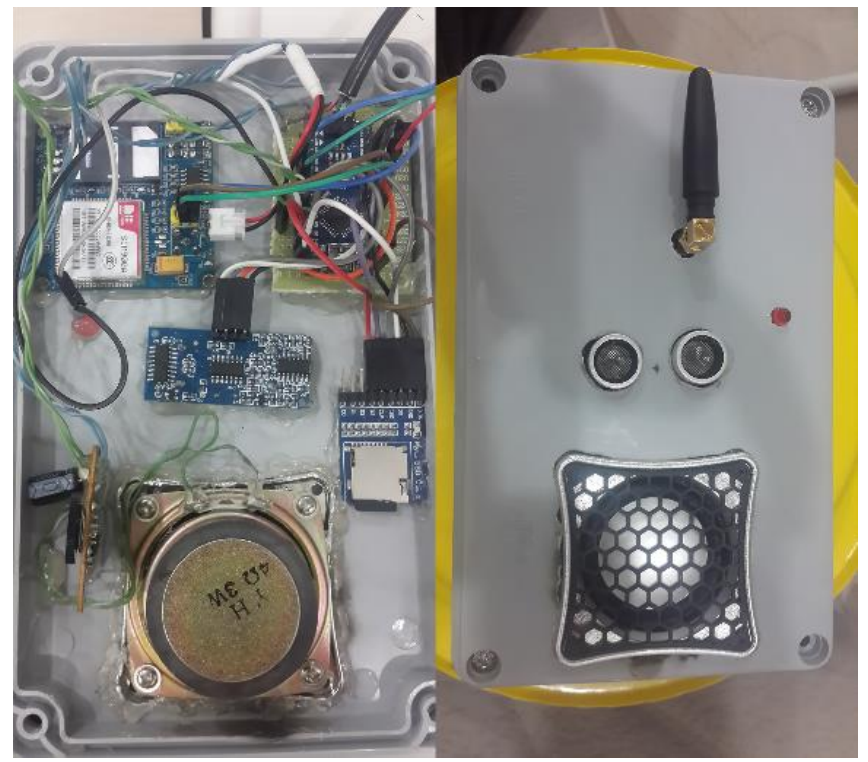

\subsection{Software}

The whole program is done using Arduino IDE. Figure 6 shows a flow chart of the Arduino program. Four libraries were used to facilities communicating with the modules. SoftwareSerial library is used to communicate with the GSM module and send AT commands to it. This library is used because of Arduino Nano does not have an additional serial port, which the GSM module rely on for communication with the microcontroller. This library transforms $\mathrm{A} 3$ and $\mathrm{A} 4$ pins into extra $\mathrm{Tx}$ and $\mathrm{Rx}$ pins to connect the $\mathrm{Tx} / \mathrm{Rx}$ pins of the GSM module. SPI library is used for communicating with the MicroSD module which depends on Serial Peripheral Interface (SPI) data protocol. SdFat library is used to manage data and read/write files on the MicroSD card. The last library is TMRpcm, which is used to output the WAV file, stored on the MicroSD, as PWM signal to digital pin 9 that connected to the speaker. The Setup function of the Arduino program first defines the used pins as outputs or inputs, then sends AT commands to the GSM module to enable text mode, enable local time/date stamp and store current settings on the GSM module memory. The time/date stamp will be stored on CSV files during logging events on. This function is also used to get the phone number and SMS alert text, which will be sent to that number in case of fullness event, from text files on the MicroSD card. This step is done to simplify changing these parameters without altering the program. The final step of Setup function is initializing digital pin 3 as an interrupt pin to connect the output pin of the PIR module to it. When the voltage rises on the output pin of the PIR module, the work of the microcontroller will be interrupted to play the WAV file on the speaker and log the usage event with the current time/date stamp on the CSV file.

The Loop function is used to measure the distance between the ultrasonic module and the trash every 15 minutes. This time period can be changed hereafter to correspond with real life operating cases. A while loop will iterate while the measured distance is smaller than a threshold, which is measured $10 \mathrm{~cm}$ according to the bin dimensions. The LED will blink for 5 seconds before a second measuring is taken inside the while loop to check if the measured value still satisfies the condition and no SMS alert has been sent. After that, the SMS alert message will be sent and the fullness event will be logged in the CSV file. Finally, as bookkeeping measure, the balance of the used SIM card is added to the SMS text before sending it. The balance is obtained by sending Unstructured Supplementary Service Data (USSD) code to the mobile network using AT command, which is executed by the GSM module. 


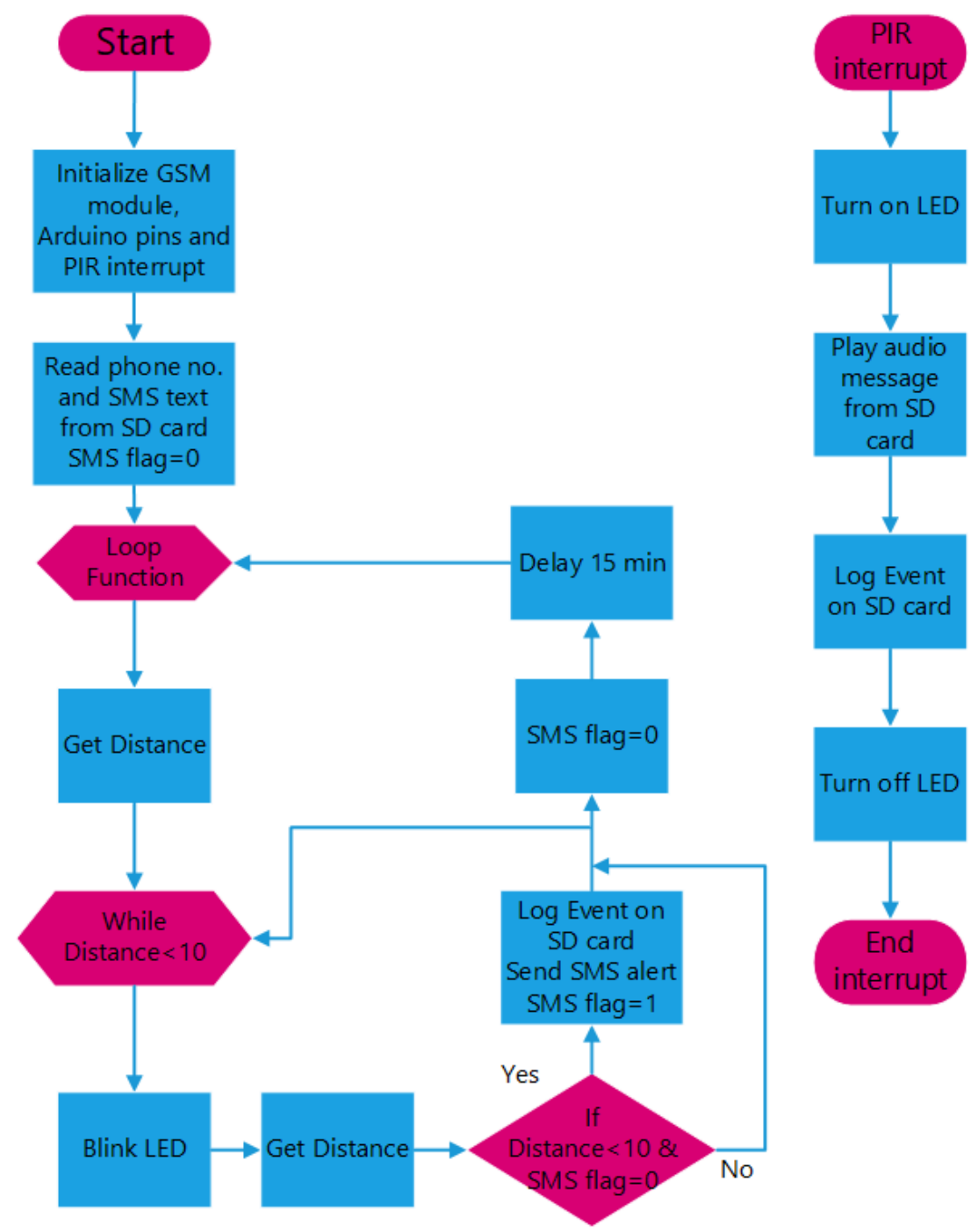

Fig 6: Software flow chart

\section{Results and Discussion}

The smart bin was tested first indoor without charging the power bank by the solar cell panel. The system worked as intended for it to do. Then the bin was installed outdoor in the main square of Nawroz University campus for a period of seven days. During this period, the solar cell panel managed successfully to charge the power bank and kept the system running. However, after examining the CSV files on the MicroSD card, the realization was that the PIR sensor kept going off and interrupt the Arduino board to play the audio message. The main reasons behind this behaviour are heat exposure and reflected sunlight from objects inside or around the bin, even though the PIR sensor datasheet points that the operating temperature of the sensor is between $-30 \mathrm{O} \mathrm{C}$ to $+70 \mathrm{O} \mathrm{C}$. The data sheet also noted that light and wind flow can be considered as interference sources. Therefore, a second outdoor test was done for another seven days with the PIR sensor is disabled. Despite that, the bin did not get full during this period but the system sent SMS fullness message every time fullness status simulated by putting an obstacle in front of the ultrasonic sensor.

In terms of power consumption, the measured current drawn by the whole system was $400 \mathrm{~mA}$, despite that the GSM module has a power rate of $2 \mathrm{~W} / 5 \mathrm{~V}$. According to the measured current, the power bank will last for 30 hours and the solar cell panel will require another 30 hours to fully charge the power bank. This is can be feasible during summer long days, as shown in the first test. However, the GSM module has a sleep mode which reduces the current consumption of the module to $1.5 \mathrm{~mA}$ during the idle period. This mode could not be implemented because of the power bank is designed to be automatically turned off when the power consumption is too low.

In terms of cost, the mobile network subscription was found satisfactory for giving 100 SMS message per 5000IQD credit. However, most mobile network companies put a 90-day expiration period on the credit. Table 1 shows the overall cost of the system without the cost of the bin itself, because of the system can be applied 
to almost any type of trash bin. If the PIR sensor and speaker were considered as an accessory, the bare minimum cost for the system will be $\$ 160$.

Finally, the solution provided by this system can be effective in managing large numbers of trash bins over a small-scale location, due to the no need for internet connectivity and computer to track the status of the bins. However, keeping aware of the bins locations according to their IDs, which are sent by the fullness SMS message, is required for a successful trash collection.

Table 1: Overall cost of the system

\begin{tabular}{|c|c|c|}
\hline Component & Fixed cost & Periodic cost \\
\hline Metal work and paint & $\$ 25$ & \\
\hline Solar panel & $\$ 34$ & \\
\hline Power bank & $\$ 30$ & \\
\hline Electric junction box & $\$ 4$ & \\
\hline Arduino Nano & $\$ 12$ & \\
\hline GSM module & $\$ 30$ & \\
\hline Ultrasonic sensor & $\$ 8$ & \\
\hline PIR sensor & $\$ 6$ & \\
\hline MicroSD card module & $\$ 5$ & \\
\hline Other parts and soldering & $\$ 10$ & \\
\hline GSM network credit & & $5000 \mathrm{IQD} \approx \$ 4$ \\
\hline
\end{tabular}

\section{Conclusion and Future Work}

Most of the past work on this subject focused on utilizing cellular network to connect to the internet for sending the sensor's data to a server. On the contrary, this paper considered using the cellular network to send fullness SMS alert directly to the user. Therefore, the work in this paper can be considered as a smart device, not as IoT solution. This system does not offer all the facilities that provided by the web applications of IoT products and papers mentioned above. However, the reduction in cost offered by this paper is noticeable, if compared with the cost of commercial products and the work in [8] due to opting out the presence of an online server. The results of the indoor test indicate that the setup worked perfectly under normal conditions. Moreover, the outdoor tests showed that solar cell panel performed adequately in charging the power bank and keeping the system running. In terms of mobile network subscription expiration period, the system can make use of postpaid plan to overcome this issue. Moreover, a custom-built power bank is recommended, with the use of USB DC-DC stepup module, Li-Ion battery charging module and $3.7 \mathrm{~V} \mathrm{Li}-$ Ion rechargeable batteries, to overcome the issue of the automatic shutdown of the power bank. This will also reduce the cost of the system by $\$ 20$. The option of charging any electronic device for the bin user can be omitted to reduce cost and receive a better performance from the power bank. Moreover, can make use of the concept of gateway or control station that mentioned in [5] and [6] to further reduce the overall cost. However, a number of bins connected to a single control station and range limitation of the Wi-Fi module must be taken into consideration.

In terms of security, the author considers it is unpractical to add accelerometer sensor to send an alert in case of vandalizing and GPS module to track the bin location in case of theft. Accelerometer sensor cannot differentiate between an animal, a person or extreme weather shaking the bin. Moreover, the first thing a thief would do is disconnecting the power from the system, thus the GPS module will be useless in tracking the bin location. However, a temperature or smoke sensor can be added to the system to send an alert in case of fire. The advantages and disadvantages of this work are illustrated in the table below 
Table 2: Advantage and Disadvantages of the System

Advantages
Low building cost.
Low operating cost.
Can work outdoors and indoors.
Can run for a long time.
Low maintenance requirements.
User-friendly design.
It has an AUX USB port to charge external electronic
devices.

Advantages

Low operating cost.

Can work outdoors and indoors.

Can run for a long time.

Low maintenance requirements.

User-friendly design.

devices.

Finally, this paper managed to present a cost-effective and user-friendly smart waste container for small-scale cases, comparing to the past work mentioned above. As a future work, the overall cost can be further reduced if the GSM module is replaced by Wi-Fi module, which connects to an intranet WLAN to send the fullness alert to the user mobile phone. This will remove the periodic cost of mobile network subscription and reduce the power consumption of the system.

\section{REFERENCES}

[1] B. Chowdhury and M. U. Chowdhury, "RFID-based Real-time Smart Waste Management System," in Australasian Telecommunication Networks and Applications Conference, 2007, no. December, pp. 175180.

[2] A. Zanella, N. Bui, A. Castellani, L. Vangelista, and M. Zorzi, "Internet of Things for Smart Cities," IEEE Internet Things J., vol. 1, no. 1, pp. 22-32, 2014.

[3] F. Mattern, "From smart devices to smart everyday objects," Proc. Smart Objects Conf., no. April, pp. 15-16, 2003.

[4] BigBellySolar, "CNN - Solar Powered Trash Compactors," 2010. [Online]. Available: https://www.youtube.com/watch?v=8e8Be9rq_C8.

[5] S. Zavare, R. Parashare, S. Patil, P. Rathod, and P. V. Babanne, "Smart City Waste Management System Using GSM," Int. J. Comput. Sci. Trends Technol., vol. 5, no. 3, pp. 74-78, 2017.

[6] T. Singh, R. Mahajan, and D. Bagai, "Smart Waste Management using Wireless Sensor Network," Int. J. Innov. Res. Comput. Commun. Eng., vol. 4, no. 6, pp. 10343-10347, 2016.

[7] S. S. Navghane, M. S. Killedar, and V. M. Rohokale, "IoT Based Smart Garbage and Waste Collection Bin," Int. J. Adv. Res. Electron. Commun. Eng., vol. 5, no. 5, pp. 1576-1578, 2016.

[8] T. Ambrose, C. Ford, and M. Norris, "Smart City Trash Cans," California Polytechnic State University, 2015.
Disadvantages

Requires manual recharging of mobile network subscription.

Requires pre-knowledge of the bins' locations.

Security measures depend on build quality and fixed installation of the bin.

Don't send an alert in case of fire.

[9] Ecube Labs, "Smart Waste Management System | Waste Analytics | Ecube Labs." [Online]. Available: http:// ecubelabs.com/.

[10] Bigbelly, "Bigbelly - Smart City Solutions." [Online]. Available: http:// bigbelly.com/.

[11]Enevo, "Waste and Recycling Services Right-Sized For You | Enevo." [Online]. Available: http://www.enevo.com/.

[12]Smart Bin, "Smartbin |Smart City|Smart Monitoring|Ultrasonic Level Sensor." [Online]. Available: https://www.smartbin.com/.

[13] Compology, "Compology | Container Monitoring For Waste." [Online]. Available: https://compology.com/.

[14]J. P. Charlesworth and J. A. G. Temple, Engineering applications of ultrasonic time-of-flight diffraction, 2nd ed. Exeter, UK: Research studies press Ltd., 1989. 